\title{
SITUACIÓN ACTUAL DE LAS ORGANIZACIONES E INSTRUMENTOS DE PROMOCIÓN DE LA INTERNACIONALIZACIÓN: UNA GUÍA PARA LAS EMPRESAS
}

\section{CURRENT STATUS OF ORGANIZATIONS AND ITS INSTRUMENTS TO PROMOTE BUSINESS INTERNATIONALIZATION: A GUIDE FOR COMPANIES}

Orihuela Orellana, Jorge (Universidad de Sevilla) *

Acedo González, Francisco José (Universidad de Sevilla)

\section{RESUMEN}

Tanto las empresas como los gobiernos han mostrado un claro interés en el fomento de la internacionalización. Pero no todas las empresas están capacitadas para acometer este reto con éxito, de forma autónoma. Las Organizaciones de Promoción de la Internacionalización (en adelante OPIs) son importantes en facilitar dichos resultados. No obstante, el problema es el desconocimiento general de las empresas en el rol y servicios ofrecidos por estas instituciones en aras de su internacionalización. Nuestro objetivo es mostrar a las empresas: quien les ayuda, las distintas OPIs que existen a disposición de las empresas y como subobjetivos dar una visión general de los servicios que prestan, así como la utilidad que representan para las empresas. Para ello hemos analizado las principales instituciones de apoyo a la Internacionalización: supranacionales, nacionales y regionales referidas a España y los instrumentos que ofrecen. El trabajo trata de sistematizar los servicios prestados y el beneficio que representan para las empresas. Así mismo se muestran instituciones de otros países, haciendo ver que todas las empresas, sin importar su ubicación, pueden recibir apoyos para su internacionalización.

Palabras claves: Promoción exportación, internacionalización, evaluación del impacto, programas. JEL: F10, F13.

\section{ABSTRACT}

Both, companies and governments, have shown a clear interest in promoting internationalization, but not all companies are qualified to undertake this challenge successfully. Organizations Promotion of Internationalization are necessary to facilitate these results. However, the problem is the general lack of knowledge shown by the companies to be aware of the role and services offered by the institutions for its internationalization. Our goal is to show institutions who helps companies to go abroad, as sub-goals to give an overview of the services provided by them to help companies. So we have analyzed the main institutions

* joriore@gmail.com

* Facultad de Ciencias Económicas y Empresariales, Departamento de Administración de Empresas y Comercialización e Investigación de Mercados (Marketing), Avda. de Ramón y Cajal, 1, 41018, Sevilla. fjacedo@us.es

Recibido: Febrero de 2016. Aceptado: Junio de 2016. 
that support single companies internationalization: supranational, national and regional referring to Spain and those instruments that they offer. This article work is usefull to systematize the services they offer and the benefit they represent for businesses. Likewise some institutions that provides this services in other countries are shown, regardless of their location all companies can receive support for internationalization.

Key words: Export promotion, internationalization, impact evaluation, programs. JEL: F10, F13.

\section{INTRODUCCIÓN}

El actual grado de internacionalización de la economía y el nivel de interrelaciones económicas entre los países hacen que, hoy en día, la globalización no sea un escenario exclusivo de las grandes empresas, sino que afecta a todos los agentes económicos. No obstante, las empresas no están solas en ese proceso, que no es tan simple como se sugiere en muchos ámbitos.

Desde el ámbito académico, se ha puesto de manifiesto la importancia de la evaluación y mejora de las estrategias que los gobiernos ponen a disposición de las empresas con el objeto de promocionar sus exportaciones (Czinkota, 2002; Griffith, Cavusgil and Xu, 2008). Este hecho también ha sido considerado desde el sector público, donde se han puesto en marcha una serie de políticas y acciones, con objeto de crear un puente entre las empresas y los mercados internacionales, de modo que se reduzca la brecha existente entre la capacidad que tiene una empresa nacional en colocar sus productos en un país extranjero frente a su competidor local en dicho mercado (Wilkinson, 2006).

El resultado de esta concienciación ha sido la puesta en marcha, tanto en países desarrollados como en países en vías de desarrollo, de organizaciones cuyo objeto es el de canalizar y desarrollar estas acciones de promoción de las exportaciones de empresas. Dichas organizaciones tienen la misión de facilitar el acceso a otros países, de modo que las empresas nacionales superen las diferentes barreras que encontrarán a la hora de colocar sus productos y servicios en dichos países-mercados. Entre los principales objetivos de estas organizaciones de promoción se encuentran los de realizar una prospección de los diferentes mercados exteriores con objeto de identificar oportunidades de negocio para sus empresas, atraer inversores extranjeros, o proveer a las empresas de su país una serie de servicios centrados en completar las carencias de éstas en materia de información, formación y asistencia técnica, de modo que puedan vender, establecerse o desarrollar un negocio ya existente, en países o regiones fuera de sus fronteras (Brossard, 1998).

En este contexto, el papel que asumen las organizaciones de promoción de la internacionalización es vital para las pequeñas y medianas empresas, debido a que su competitividad local se ve seriamente amenazada por empresas extranjeras que acceden a su mercado natural y unido a su baja capacidad para acceder a mercados exteriores hace que encuentren serios problemas para subsistir en el mercado nacional. En este sentido, el apoyo que reciben de las organizaciones de promoción es clave para ellas.

\section{LA PERSPECTIVA ACADÉMICA}

A medida que los estados han ido dando importancia a estas organizaciones de promoción de la internacionalización, el ámbito académico se ha planteado diversas cuestiones sobre: su impacto, funcionamiento o incluso función. Sirva a modo de resumen el 
siguiente cuadro donde se muestran los trabajos sobre el desarrollo teórico y metodológico que se ha publicado en relación al apoyo que las instituciones de fomento de la internacionalización realizan a las empresas.

\section{CUADRO 1: PRINCIPALES TRABAJOS SOBRE LAS OPIS}

\begin{tabular}{|c|c|c|c|}
\hline Titulo artículo & Autor & Revista & Año \\
\hline $\begin{array}{l}\text { Insights into public export promotion } \\
\text { programs in an emerging economy: The case } \\
\text { of Malaysian SMEs }\end{array}$ & $\begin{array}{l}\text { Ayob y } \\
\text { Freixanet }\end{array}$ & Evaluation and Program Planning & 2014 \\
\hline $\begin{array}{l}\text { Speed of the internationalization process: The } \\
\text { role of diversity and depth in experiential } \\
\text { learning }\end{array}$ & $\begin{array}{l}\text { Casillas y } \\
\text { Moreno- } \\
\text { Menéndez }\end{array}$ & $\begin{array}{c}\text { Journal of International Business } \\
\text { Studies }\end{array}$ & 2014 \\
\hline Does location matter to export performance? & $\begin{array}{l}\text { Freeman y } \\
\text { Styles }\end{array}$ & International Marketing Review & 2014 \\
\hline $\begin{array}{l}\text { Do export promotion agencies increase } \\
\text { exports? }\end{array}$ & $\begin{array}{l}\text { Hayakawa, Lee } \\
\text { y Park }\end{array}$ & Developing Economies & 2014 \\
\hline $\begin{array}{l}\text { An economic evaluation of public programs } \\
\text { for internationalization: The case of the } \\
\text { Diagnostic program in Spain }\end{array}$ & $\begin{array}{l}\text { Cansino, Lopez- } \\
\text { Melendo, Pablo- } \\
\text { Romero, y } \\
\text { Sánchez-Braza }\end{array}$ & Evaluation and Program Planning & 2013 \\
\hline $\begin{array}{l}\text { Export promotion organization emergence } \\
\text { and development: a call to research }\end{array}$ & $\begin{array}{l}\text { Gillespie y } \\
\text { Riddle }\end{array}$ & International Marketing Review & 2004 \\
\hline $\begin{array}{l}\text { Understanding the role of export marketing } \\
\text { assistance: empirical evidence and research } \\
\text { needs. }\end{array}$ & $\begin{array}{l}\text { Diamantopoulos, } \\
\text { Schlegelmilch, y } \\
\text { Tse }\end{array}$ & European Journal of Marketing & 1993 \\
\hline $\begin{array}{l}\text { The role of informational assistance in small } \\
\text { firms }\end{array}$ & Seringhaus & International Marketing Review & 1987 \\
\hline $\begin{array}{l}\text { A pilot evaluation of the export marketing } \\
\text { research scheme }\end{array}$ & Lemaghen & $\begin{array}{c}\text { British Overseas Trade Board, } \\
\text { Schanmark Export }\end{array}$ & 1987 \\
\hline $\begin{array}{l}\text { The impact of government export marketing } \\
\text { assistance }\end{array}$ & Seringhaus & International Marketing Review & 1986 \\
\hline $\begin{array}{l}\text { The role of marketing incentives in export } \\
\text { promotion: the Uruguayan case }\end{array}$ & Brezzo y Perkal & Export Promotion & 1983 \\
\hline Export development strategies & Czinkota & Praeger & 1982 \\
\hline
\end{tabular}

El trabajo de Gillespie y Riddle (2004) muestra de forma detallada el papel y las actividades desarrollados desde estas instituciones, como se desprende de la figura 1.

Así, las organizaciones de promoción a la internacionalización OPI (EPO- Export Promotion Organizations en inglés), creadas por los gobiernos, comienzan definiendo una estrategia para la internacionalización de sus empresas, a continuación ofrecen unos instrumentos de apoyo a a las empresas con las que trabajan y adaptados a sus necesidades. En tercer lugar, estas instituciones informan a las empresas de la existencia de estos instrumentos, realizando acciones de información y comunicación. Finalmente, las empresas usan los servicios ofrecidos y obtienen una mejora de sus resultados en la exportación. 


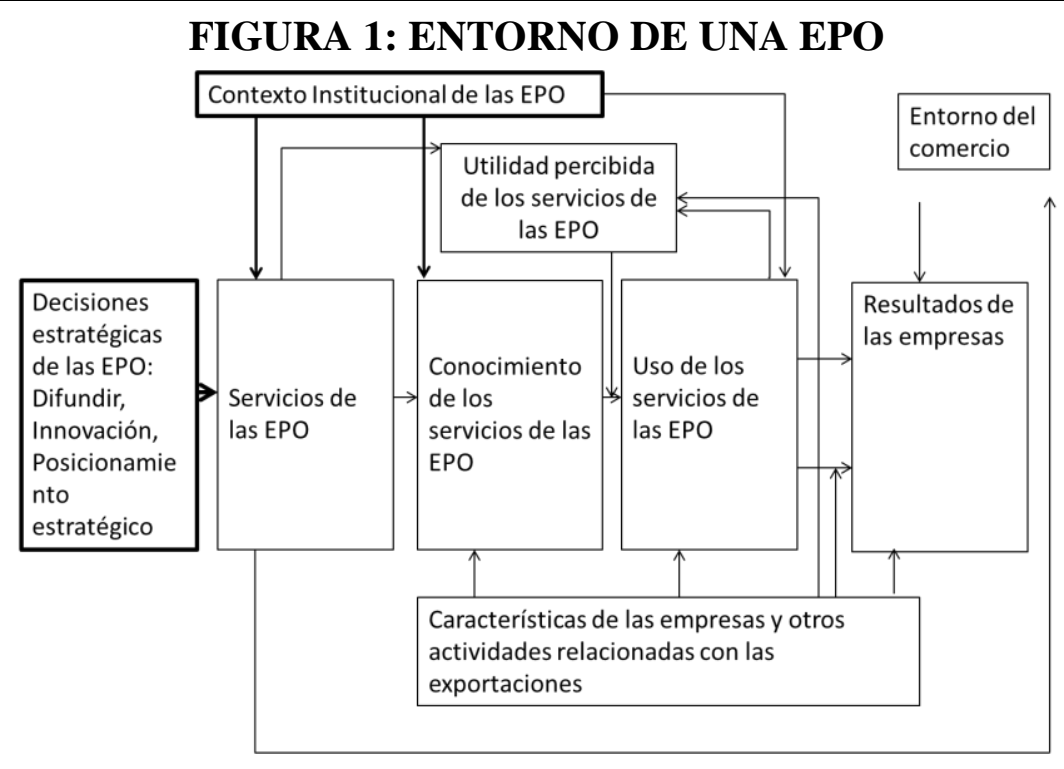

Fuente: Gillespie y Riddle, 2004

En definitiva, este es el proceso teórico o conceptual que desea realizar cualquier organización de promoción de la internacionalización, con objeto de la internacionalización de sus empresas.

En la práctica, el proceso de apoyo a la internacionalización se inicia de la siguiente manera: cada organización hace un análisis de las necesidades en internacionalización que tienen las empresas en su área de competencia, poniendo a su disposición los instrumentos que demandan o precisen. No obstante, no todas las instituciones son idénticas en su configuración ni funcionamiento. Su estructura e instrumentos ofrecidos a las empresas pueden ser el resultado de la innovación por parte de los fundadores y líderes de estas instituciones (Aldrich, 1999) y, aunque no es posible sistematizar la casuística ni el uso que hacen las empresas de los instrumentos, sí se puede realizar una categorización de las organizaciones y los instrumentos de apoyo a la internacionalización, a fin de facilitar su comprensión.

\section{METODOLOGÍA}

Se ha empleado un método descriptivo, dando la visión de las distintas instituciones de apoyo a la internacionalización que ellos quieren dar a los usuarios y para ello hemos usado como fuente de datos la información que ofrecen en sus páginas webs con respecto a los servicios que ofrecen.

También se ha empleado información ofrecida por las distintas instituciones organismos nacionales o supranacionales.

\section{CLASIFICACIÓN DE LAS ORGANIZACIONES DE PROMOCIÓN DE LA INTERNACIONALIZACIÓN}

En el presente epígrafe centraremos la atención en todos aquellos organismos nacionales e internacionales comprometidos con la promoción de la internacionalización de las empresas españolas, determinando el papel que desempeñan, así como su grado de coordinación e integración. 


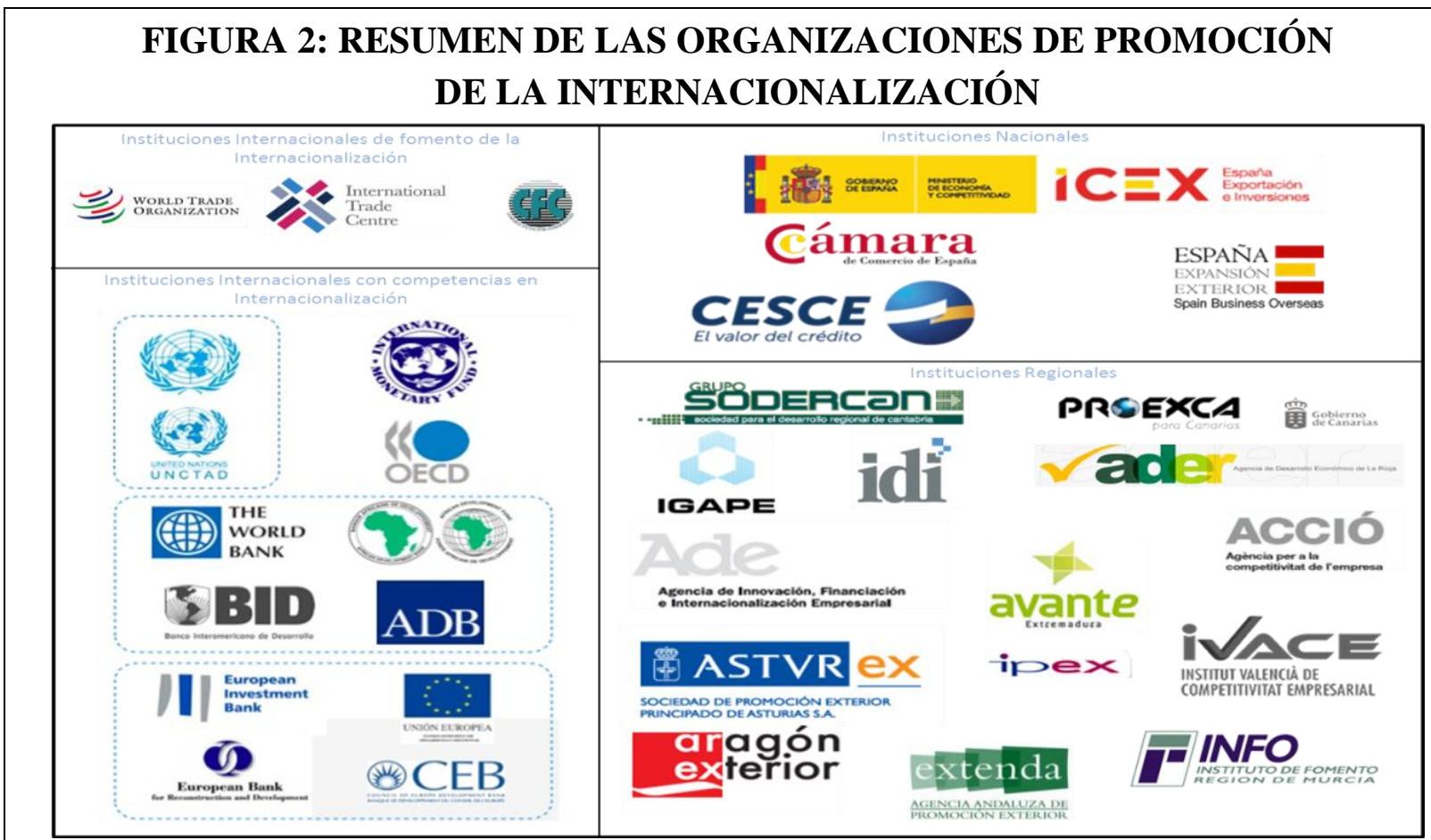

Fuente: Elaboración propia

Estos organismos e instituciones son los que diseñan, desarrollan y ejecutan las políticas públicas enfocadas en la promoción de la internacionalización. En virtud del análisis realizado sobre las mismas, y que a continuación exponemos, se ponen de manifiesto cuáles son las bases para la identificación de las necesidades que satisfacen las organizaciones de promoción de la internacionalización.

Se van a clasificar las instituciones en función de su ámbito geográfico de actuación, lo que permitirá una visión de conjunto acorde con la realidad. Según este criterio clasificaremos las instituciones en los siguientes grupos: internacionales, nacionales y regionales, tal y como se ven en la figura 2.

Para cada uno de estos tres grupos analizaremos las instituciones que se dedican en exclusiva a realizar acciones de promoción de la internacionalización, así como aquellas instituciones que realizan acciones ocasionales en este ámbito o cuyas actuaciones tienen un impacto indirecto en el sistema de promoción de la internacionalización.

Sin pretender profundizar en cada una de ellas se mostrará las áreas donde presta servicios y sus principales características. Se analizan las áreas de información o asesoramiento, si realiza publicaciones, los medios de contacto, los instrumentos que ofrece y la evaluación de sus resultados que realiza. En concretos se van a analizar un total de 33 instituciones de fomento a la internacionalización (con dedicación parcial o total) con especial interés para las empresas españolas.

A fin de facilitar la lectura, hemos sintetizado la información en tablas donde se recogen todos estos elementos, así como si suponen algún coste para las empresas.

No obstante, no son todas, y existen un gran número de organizaciones con el mismo fin en todo el planeta. Sirva como muestra la página web de Papeles de Inteligencia ${ }^{1}$, en la que se

\footnotetext{
${ }^{1}$ http://papelesdeinteligencia.com/institutos-de-comercio-internacional-y-exportacion/
} 
muestran 39 instituciones de apoyo a la internacionalización centrales de 39 países, clasificados por áreas geográficas, y que se muestran a continuación.

Esta información sólo se ofrece para aquellas empresas o instituciones que quieran analizar o usar los servicios que se prestan en otros países.

\begin{tabular}{|l|}
\hline Europa \\
\hline Alemania: http://www.bfai.de/ \\
\hline Bélgica: http://www.abh-ace.be/ \\
\hline España: http://www.icex.es/ \\
\hline Francia: http://www.ubifrance.fr/default.html \\
\hline Grecia: http://www.hepo.gr/ \\
\hline Irlanda: http://www.enterprise-ireland.com/en/ \\
\hline Israel: http://www.export.gov.il// \\
\hline Italia: http://www.ice.gov.it/ \\
\hline Noruega: http://www.nortrade.com/ \\
\hline Portugal: http://www.portugalglobal.pt/PT/Paginas/Home.aspx \\
\hline Reino Unido: http://www.ukti.gov.uk/es_es/home.html? \\
\hline América \\
\hline Argentina: http://www.exportar.org.ar/ \\
\hline Brasil: http://www.portaldoexportador.gov.br/ \\
\hline Bolivia: http://www.ibce.org.bo/ \\
\hline Canadá: http://www.dfait-maeci.gc.ca/ \\
\hline Colombia: http://www.proexport.com.co/ \\
\hline Chile: http://www.prochile.cl// \\
\hline Estados Unidos: http://www.ita.doc.gov/ \\
\hline México: http://www.bancomext.gob.mx/Bancomext/secciones/home.html \\
\hline Panamá: http://www.mici.gob.pa/base.php?hoja=homepage \\
\hline Perú: http://www.promperu.gob.pe/ \\
\hline Asia y Oceanía \\
\hline Australia: http://www.austrade.gov.au/ \\
\hline China: http://english.mofcom.gov.cn/ \\
\hline Corea del sur: http://www.kotra.or.kr/wps/portal/dknew \\
\hline Filipinas: http://www.citem.com.ph/ \\
\hline Hong Kong: http://www.hktdc.com/ \\
\hline India: http://www.fieo.org/ \\
\hline Indonesia: http://www.nafed.go.id/ \\
\hline Malasia:http://www.matrade.gov.my/ \\
\hline Nueva Zelanda: http://www.nzte.govt.nz/Pages/default.aspx \\
\hline Japón: http://www.jetro.go.jp/ \\
\hline Singapur: http://www.iesingapore.gov.sg/wps/portal \\
\hline Tailandia: http://www.thaitrade.com/home \\
\hline Vietnam: http://www.vietrade.gov.vn/ \\
\hline África y Oriente Medio \\
\hline Argelia: http://www.promex.dz/ \\
\hline Omán: http://www.ociped.com// \\
\hline Túnez: http://www.cepex.nat.tn/content/accueil \\
\hline Turquía: http://www.ibp.gov.tr/ \\
\hline Sudáfrica: http://www.wesgro.org.za/ \\
\hline
\end{tabular}


A continuación se ofrecen unos cuadros que muestran los servicios ofrecidos por OPI desde los niveles supranacional, nacional y regional:

Supranacional:

- OMC: Organización Mundial del Comercio

- ITC: International Trade Center

- CFC: Common Fund for Commodities.

- IFMs: Instituciones Financieras Multilaterales

Nacionales:

- ICEX: Instituto de Comercio Exterior

- Secretaría de Estado de Comercio

- La Cámara: Cámaras de Comercio de España

- CESCE: Compañía Española de Seguro de Crédito a la Exportación

- Sociedad Estatal España Expansión Exterior

Regionales:

- Acció Exterior: Cataluña

- IPEX: Castilla la Mancha

- Extenda-Agencia Andaluza de Promoción Exterior SA.

- Avante Extremadura

- La Rioja: ADER

- IGAPE Instituto Gallego de Promoción Exterior

- SODERCAN: Sociedad Desarrollo de Cantabria

- PROEXCA: Promoción Exterior de Canarias-

- ADE EXCAL: Castilla León

- IVACE: Instituto Valenciano de Comercio Exterior

- INFO Murcia

- Asturias: ASTUREX

- Aragón Exterior.

- IDI Baleares 


\begin{tabular}{|c|c|c|c|c|c|c|c|c|c|c|c|c|c|}
\hline \multicolumn{14}{|c|}{$\begin{array}{c}\text { CUADRO 2: INSTITUCIONES Y ORGANISMOS DE PROMOCIÓN DE LA INTERNACIONALIZACIÓN } \\
\text { A NIVEL INTERNACIONAL }\end{array}$} \\
\hline \multicolumn{2}{|r|}{ ORGANISMO } & \multicolumn{3}{|c|}{ OMC } & \multicolumn{3}{|c|}{ ITC } & \multicolumn{3}{|c|}{ CFC } & \multicolumn{3}{|c|}{ IFMs } \\
\hline \multirow{18}{*}{$\begin{array}{l}\text { AREAS DE } \\
\text { INFORMACIÓN I } \\
\text { ASESORAMIENTO }\end{array}$} & Tipo de información o asesoramiento & $\begin{array}{c}\text { Info. } \\
\text { gratuita }\end{array}$ & $\begin{array}{l}\text { Asesora. } \\
\text { Gratuito }\end{array}$ & $\begin{array}{l}\text { Asesora. } \\
\text { de Pago }\end{array}$ & $\begin{array}{c}\text { Info. } \\
\text { gratuita }\end{array}$ & $\begin{array}{c}\text { Asesora. } \\
\text { Gratuito }\end{array}$ & $\begin{array}{l}\text { Asesora. } \\
\text { de Pago }\end{array}$ & $\begin{array}{c}\text { Info. } \\
\text { gratuita }\end{array}$ & $\begin{array}{c}\text { Asesora. } \\
\text { Gratuito }\end{array}$ & $\begin{array}{l}\text { Asesora. } \\
\text { de Pago }\end{array}$ & $\begin{array}{c}\text { Info. } \\
\text { gratuita }\end{array}$ & $\begin{array}{l}\text { Asesora. } \\
\text { Gratuito }\end{array}$ & $\begin{array}{l}\text { Asesora. } \\
\text { de Pago }\end{array}$ \\
\hline & \begin{tabular}{|l|} 
Información pais \\
\end{tabular} & $\mathrm{x}$ & & & $x$ & & & $\mathrm{x}$ & & & $\mathrm{x}$ & $\mathrm{x}$ & \\
\hline & Para exportar & $\mathrm{x}$ & & & $\mathrm{x}$ & & $\mathrm{x}$ & $\mathrm{x}$ & & & $\mathrm{x}$ & $\mathrm{x}$ & \\
\hline & \begin{tabular}{|l} 
Para invertir en el exterior \\
\end{tabular} & $x$ & & & $x$ & & $x$ & $x$ & & & $x$ & $x$ & \\
\hline & Estadísticas & $\mathrm{x}$ & & & $\mathrm{x}$ & & & $\mathrm{x}$ & & & $\mathrm{x}$ & & \\
\hline & Contactos comerciales & & & & & & $x$ & & & $\mathrm{x}$ & $x$ & & $x$ \\
\hline & Informes económicos de empresas & & & & & & $\mathrm{x}$ & & & & & & \\
\hline & Oportunidades de negocio & & & & $x$ & & & $\mathrm{x}$ & & & $x$ & & \\
\hline & Contratación y fiscalidad internacional & $\mathrm{x}$ & & & $\mathrm{x}$ & & $\mathrm{x}$ & $\mathrm{x}$ & & & $\mathrm{x}$ & $\mathrm{x}$ & \\
\hline & Información sobre temas comunitarios & $\mathrm{x}$ & & & $\mathrm{x}$ & & & & & & $\mathrm{x}$ & $\mathrm{x}$ & \\
\hline & \begin{tabular}{|l} 
Apoyo Logístico en el exterior \\
\end{tabular} & & & & $\mathrm{x}$ & & $\mathrm{x}$ & $\mathrm{x}$ & & & & $\mathrm{x}$ & \\
\hline & Información sobre programas & & & & $\mathrm{x}$ & & & $\mathrm{x}$ & & & $\mathrm{x}$ & & \\
\hline & Operativa de Comercio Exterior & & & & $x$ & & $\mathrm{x}$ & & & $\mathrm{x}$ & $\mathrm{x}$ & $\mathrm{x}$ & \\
\hline & Estrategias internacionalización & & & & $x$ & & & & & & $\mathrm{x}$ & & \\
\hline & \begin{tabular}{|l|} 
Política comercial \\
\end{tabular} & $\mathrm{x}$ & & & $x$ & & & $\mathrm{x}$ & & & $x$ & & \\
\hline & Información para empresas extranjeras & & & & $\mathrm{x}$ & & & & & & $\mathrm{x}$ & & \\
\hline & $1+D+i$ & & & & $x$ & & & & & & $x$ & & \\
\hline & $\mid \begin{array}{l}\text { Inteligencia Competitiva } \\
\end{array}$ & & & & $\bar{x}$ & & & & & & $\bar{x}$ & & \\
\hline \multirow{2}{*}{\multicolumn{2}{|c|}{\begin{tabular}{|l} 
PUBLICACIONES \\
PRODUCTOS ESPECIFICOS \\
\end{tabular}}} & \multicolumn{3}{|c|}{$\bar{x}$} & \multicolumn{3}{|c|}{$\bar{x}$} & \multicolumn{3}{|c|}{$\bar{x}$} & \multirow{2}{*}{\multicolumn{3}{|c|}{$\frac{x}{x}$}} \\
\hline & ECIFICOS & & & & \multirow{2}{*}{\multicolumn{3}{|c|}{$\frac{x}{x}$}} & \multicolumn{3}{|c|}{$\frac{x}{x}$} & & & \\
\hline \multicolumn{2}{|c|}{ PAISES } & \multicolumn{3}{|c|}{$\bar{x}$} & & & & \multirow{2}{*}{\multicolumn{3}{|c|}{$\frac{x}{x}$}} & \multicolumn{3}{|c|}{$\frac{x}{x}$} \\
\hline \multicolumn{2}{|l|}{ SECTORES } & \multicolumn{3}{|c|}{$x$} & \multicolumn{3}{|c|}{$x$} & & & & \multicolumn{3}{|c|}{$\bar{x}$} \\
\hline \multirow{5}{*}{$\begin{array}{l}\text { MEDIOS DE } \\
\text { CONTACTO }\end{array}$} & Teléfono & & sí & & & si & & & sí & & & si & \\
\hline & Correo Electrónico & & sí & & & si & & & sí & & & si & \\
\hline & Web (formularios) & & si & & & si & & & si & & & si & \\
\hline & Presencial & & sí & & & si & & & sí & & & si & \\
\hline & Redes sociales & & sí & & & si & & & & & & si & \\
\hline & Contact Center & & si & & & sí & & & sí & & & sí & \\
\hline PARALA & Sist. Informát. En la gestión de consultas & & sí & & & si & & & sí & & & si & \\
\hline GESTIÓN & CRM & & sí & & & si & & & sí & & & si & \\
\hline & Atención presencial & & sí & & & si & & & sí & & & si & \\
\hline & De forma centralizada & & sí & & & si & & & sí & & & si & \\
\hline EL SERVICIO SE & Distribuida a través de una red de oficinas & & sí & & & si & & & & & & si & \\
\hline PRESTA: & 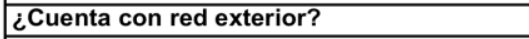 & & sí & & & si & & & & & & si & \\
\hline & Otras redes & & & & & & & & sí & & & & \\
\hline EVALUACIÓN DE & Encuesta de Satisfacción & & & & & & & & & & & sí & \\
\hline RESULTADOS & Certificación en Calidad & & sí & & & sí & & & sí & & & sí & \\
\hline
\end{tabular}




\begin{tabular}{|c|c|c|c|c|c|c|c|c|c|c|c|c|c|c|c|c|}
\hline \multicolumn{17}{|c|}{$\begin{array}{c}\text { CUADRO 3: RESUMEN DE LAS AGENCIAS DE PROMOCIÓN DE LA INTERNACIONALIZACIÓN } \\
\text { A NIVEL NACIONAL Y SUS SERVICIOS }\end{array}$} \\
\hline \multicolumn{2}{|l|}{ ORGANISMO } & \multicolumn{3}{|c|}{$\begin{array}{l}\text { Secretaría de Estado } \\
\text { de Comercio }\end{array}$} & \multicolumn{3}{|c|}{$\begin{array}{l}\text { SOCIEDAD ESTATAL } \\
\text { ESPAÑA EXPANSION } \\
\text { EXTERIOR }\end{array}$} & \multicolumn{3}{|c|}{ ICEX } & \multicolumn{3}{|c|}{ LA CAMARA } & \multicolumn{3}{|c|}{ CESCE } \\
\hline \multirow{18}{*}{$\begin{array}{l}\text { AREAS DE } \\
\text { INFORMACIÓN } / \\
\text { ASESORAMIENTO }\end{array}$} & Tipo de información o asesoramiento & \begin{tabular}{|c|} 
Info. \\
gratuita
\end{tabular} & $\begin{array}{l}\text { Asesora. } \\
\text { Gratuito }\end{array}$ & \begin{tabular}{|l|}
$\begin{array}{l}\text { Asesora. } \\
\text { de Pago }\end{array}$ \\
\end{tabular} & \begin{tabular}{|c|} 
Info. \\
gratuita
\end{tabular} & \begin{tabular}{|l|} 
Asesora. \\
Gratuito
\end{tabular} & \begin{tabular}{|l|} 
Asesora. \\
de Pago
\end{tabular} & $\begin{array}{c}\text { Info. } \\
\text { gratuita }\end{array}$ & $\begin{array}{l}\text { Asesora. } \\
\text { Gratuito }\end{array}$ & $\begin{array}{l}\text { Asesora. } \\
\text { de Pago }\end{array}$ & $\begin{array}{c}\text { Info. } \\
\text { gratuita }\end{array}$ & $\begin{array}{l}\text { Asesora. } \\
\text { Gratuito }\end{array}$ & \begin{tabular}{|l|} 
Asesora. \\
de Pago
\end{tabular} & \begin{tabular}{|c|} 
Info. \\
gratuita
\end{tabular} & \begin{tabular}{|l|} 
Asesora. \\
Gratuito
\end{tabular} & $\begin{array}{r}\text { Asesora. } \\
\text { de Pago }\end{array}$ \\
\hline & Información pais & $x$ & & & & & & $\mathrm{x}$ & & $x$ & $\mathrm{x}$ & & & $x$ & & $x$ \\
\hline & \begin{tabular}{|l|} 
Para exportar \\
\end{tabular} & $x$ & & & & & $\mathrm{x}$ & $x$ & & $\bar{x}$ & $x$ & & & & & $\bar{x}$ \\
\hline & Para invertir en el exterior & $\bar{x}$ & & & & & $\mathrm{x}$ & $\mathrm{x}$ & & $\mathrm{x}$ & & & & & & $\frac{1}{x}$ \\
\hline & Estadísticas & $\mathrm{x}$ & & & & & $\hat{n}$ & $\hat{x}$ & & $\hat{n}$ & $\mathrm{x}$ & & & $\bar{x}$ & & $\frac{x}{x}$ \\
\hline & \begin{tabular}{|l|} 
Contactos comerciales \\
\end{tabular} & $x$ & & & & & $\mathrm{x}$ & $x$ & & $\mathrm{x}$ & $\therefore$ & & $\mathrm{x}$ & 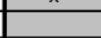 & & $\frac{x}{x}$ \\
\hline & Informes económicos de empresas & & & & & & & & & & & & $\mathrm{x}$ & & & $x$ \\
\hline & \begin{tabular}{|l|} 
Oportunidades de negocio \\
\end{tabular} & $\mathrm{x}$ & & & & & $\mathrm{x}$ & $\mathrm{x}$ & & $\mathrm{x}$ & $\mathrm{x}$ & & & & & \\
\hline & Contratación y fiscalidad internacional & $\mathrm{x}$ & & & & & $x$ & $x$ & & & $\mathrm{x}$ & & & $\mathrm{x}$ & & $x$ \\
\hline & Información sobre temas comunitarios & & & & & & & & & & $\mathrm{x}$ & & & & & \\
\hline & \begin{tabular}{|l} 
Apoyo Logistico en el exterior \\
\end{tabular} & $\mathrm{x}$ & & & & & 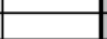 & $\mathrm{x}$ & & $\mathrm{x}$ & $\hat{x}$ & & & & & \\
\hline & Información sobre programas & $\mathrm{x}$ & & & & & & $\hat{x}$ & & & $\hat{x}$ & & & $\bar{x}$ & & \\
\hline & $\begin{array}{l}\text { Operativa de Comercio Exterior } \\
\end{array}$ & & & & & & $\mathrm{x}$ & & & $\mathrm{x}$ & $\bar{x}$ & & & & & $x$ \\
\hline & \begin{tabular}{|l|} 
Estrategias internacionalización \\
\end{tabular} & $\mathrm{x}$ & & & & & $\mathrm{x}$ & $\mathrm{x}$ & & & $x$ & & & & & \\
\hline & \begin{tabular}{|l|} 
Politica comercial \\
\end{tabular} & $\bar{x}$ & & & & & & $x$ & & & $\mathrm{x}$ & & & & & \\
\hline & Información para empresas extranjeras & $x$ & & & & & $x$ & $x$ & & & $x$ & & & & & $\bar{x}$ \\
\hline & $1+D+i$ & & & & & & & & & & & & & & & \\
\hline \multirow{2}{*}{\multicolumn{2}{|c|}{\begin{tabular}{|l}
\multicolumn{1}{|c|}{ Inteligencia Competitiva } \\
PUBLICACIONES
\end{tabular}}} & & & & & & & & & & & & $\bar{x}$ & & & \\
\hline & & & & & & & & \multicolumn{3}{|c|}{$\bar{x}$} & \multicolumn{3}{|c|}{$\bar{x}$} & \multicolumn{3}{|c|}{$\bar{x}$} \\
\hline \multirow{2}{*}{\multicolumn{2}{|c|}{$\begin{array}{l}\text { PRODUCTOS ESPECIFICOS } \\
\text { PAISES }\end{array}$}} & & & & & $\bar{x}$ & & \multirow{2}{*}{\multicolumn{3}{|c|}{$\frac{x}{x}$}} & \multirow{2}{*}{\multicolumn{3}{|c|}{$\frac{\bar{x}}{x}$}} & \multirow{2}{*}{\multicolumn{3}{|c|}{$\frac{\bar{x}}{x}$}} \\
\hline & & & & & \multirow{2}{*}{\multicolumn{3}{|c|}{$\frac{x}{x}$}} & & & & & & & & & \\
\hline \multicolumn{2}{|c|}{ SECTORES } & & & & & & & \multicolumn{3}{|c|}{$\frac{\hat{x}}{x}$} & \multicolumn{3}{|c|}{$\frac{x}{x}$} & \multicolumn{3}{|c|}{$\frac{x}{x}$} \\
\hline 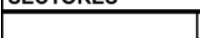 & Teléfono & & si & & & $\hat{\mathrm{si}}$ & & & $\hat{\mathrm{si}}$ & & & $\hat{\mathrm{si}}$ & & & $\hat{\mathrm{si}}$ & \\
\hline & \begin{tabular}{|l|} 
Correo Electrónico \\
\end{tabular} & & si & & & si & & & si & & & si & & & si & \\
\hline $\begin{array}{l}\text { MEDIOS DE } \\
\text { CONTACTO }\end{array}$ & Web (formularios) & & si & & & si & & & si & & & si & & & si & \\
\hline & Presencial & & si & & & si & & & si & & & si & & & si & \\
\hline & \begin{tabular}{|l|} 
Redes sociales \\
\end{tabular} & & si & & & si & & & si & & & si & & & si & \\
\hline & Contact Center & & si & & & no & & & si & & & si & & & si & \\
\hline INSTRUMENTOS & Sist. Informát. En la gestión de consultas & & si & & & si & & & si & & & si & & & si & \\
\hline PARA LA GESTIÓN & & & si & & & si & & & si & & & si & & & si & \\
\hline & \begin{tabular}{|l|} 
Atención presencial \\
\end{tabular} & & si & & & si & & & si & & & si & & & si & \\
\hline & De forma centralizada & & si & & & si & & & si & & & SI & & & si & \\
\hline EL SERVICIO SE & Distribuida a través de una red de oficinas & & si & & & si & & & si & & & SI & & & si & \\
\hline $\begin{array}{l}\text { PRESTA: } \\
\text { PRT }\end{array}$ & $\begin{array}{l}\text { ¿Cuenta con red exterior? } \\
\end{array}$ & & si & & & si & & & si & & & SI & & & si & \\
\hline & Otras redes & & & & & & & & & & & SI & & & & \\
\hline \begin{tabular}{|l|} 
EVALUACIÓN DE \\
\end{tabular} & Encuesta de Satisfacción & & si & & & & & & si & & & si & & & si & \\
\hline RESULTADOS & Certificación en Calidad & & si & & & si & & & si & & & no & & & si & \\
\hline
\end{tabular}




\begin{tabular}{|c|c|c|c|c|c|c|c|c|c|c|c|c|c|c|c|c|c|c|c|c|c|c|}
\hline \multicolumn{23}{|c|}{ CUADRO 4: INSTITUCIONES Y ORGANISMOS A NIVEL REGIONAL } \\
\hline \multicolumn{2}{|l|}{ ORGANISMO } & \multicolumn{3}{|c|}{ ACC10 } & \multicolumn{3}{|c|}{ ARAGON EXTERIOR } & \multicolumn{3}{|c|}{ ASTUREX } & \multicolumn{3}{|c|}{ EXCAL } & \multicolumn{3}{|c|}{ EXTENDA } & \multicolumn{3}{|c|}{ IDI } & \multicolumn{3}{|c|}{ IGAPE } \\
\hline \multirow{15}{*}{$\begin{array}{c}\text { AREAS DE } \\
\text { INFORMACIÓN / } \\
\text { ASESORAMIENTT }\end{array}$} & Tipo de información o asesoramiento & $\left|\begin{array}{c}\text { Info. } \\
\text { gratuita }\end{array}\right|$ & $\begin{array}{l}\text { Asesora. } \\
\text { Gratuito }\end{array}$ & $\begin{array}{l}\text { Asesora. } \\
\text { de Pago }\end{array}$ & \begin{tabular}{|c|} 
Info. \\
gratuita
\end{tabular} \mid & $\begin{array}{c}\text { Asesora. } \\
\text { Gratuito }\end{array} \mid$ & $\begin{array}{l}\text { Asesora. } \\
\text { de Pago }\end{array}$ & \begin{tabular}{|c} 
Info. \\
gratuita
\end{tabular} & $\begin{array}{l}\text { Asesora. } \\
\text { Gratuito }\end{array}$ & $\begin{array}{l}\text { Asesora. } \\
\text { de Pago }\end{array}$ & \begin{tabular}{|c|} 
Info. \\
gratuita
\end{tabular} & $\begin{array}{c}\text { Asesora. } \\
\text { Gratuito }\end{array}$ & $\begin{array}{l}\text { Asesora. } \\
\text { de Pago }\end{array}$ & $\begin{array}{c}\text { Info. } \\
\text { gratuita }\end{array}$ & $\begin{array}{c}\text { Asesora. } \\
\text { Gratuito }\end{array}$ & $\begin{array}{l}\text { Asesora. } \\
\text { de Pago }\end{array}$ & $\begin{array}{l}\text { Info. } \\
\text { gratuita }\end{array}$ & $\begin{array}{l}\text { Asesora. } \\
\text { Gratuito }\end{array}$ & $\begin{array}{c}\text { Asesora. } \\
\text { de Pago }\end{array}$ & \begin{tabular}{|c|c|c|c|} 
Info. \\
gratuita
\end{tabular} & $\begin{array}{l}\text { Asesora. } \\
\text { Gratuito }\end{array}$ & $\begin{array}{l}\text { Asesora. } \\
\text { de Pago }\end{array}$ \\
\hline & \begin{tabular}{|l} 
Información pais \\
\end{tabular} & $x$ & & $x$ & & & $x$ & $\mathrm{x}$ & & & $x$ & $x$ & & $x$ & $x$ & & $x$ & & & $x$ & $x$ & \\
\hline & Para exportar & $x$ & & $\frac{x}{x}$ & & & $\frac{x}{x}$ & & $x$ & & $\frac{x}{x}$ & $\hat{x}$ & & $\frac{x}{x}$ & $\frac{x}{x}$ & & $\mathrm{x}$ & $x$ & & $x$ & $x$ & \\
\hline & $\begin{array}{l}\text { Para invertir en el exterior } \\
\text { Tat }\end{array}$ & $\frac{x}{x}$ & $\frac{x}{x}$ & $\frac{x}{x}$ & & & $\frac{x}{x}$ & 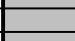 & & $x$ & $\frac{x}{x}$ & & & $\frac{x}{x}$ & $\frac{x}{x}$ & & $x$ & & & $\bar{x}$ & $x$ & \\
\hline & & $x$ & $x$ & $\frac{x}{x}$ & & & $\frac{x}{x}$ & & & & $\mathrm{x}$ & $x$ & & $x$ & $x$ & & $x$ & & & $\mathrm{x}$ & $\mathrm{x}$ & \\
\hline & \begin{tabular}{|l|} 
Contactos comerciales \\
Informes económicos de empresas
\end{tabular} & $\mathrm{x}$ & & $\frac{x}{x}$ & & & & & & $x$ & $x$ & $\frac{x}{x}$ & $\mathrm{Y}$ & $x$ & $x$ & & $\mathrm{x}$ & $x$ & & $\bar{x}$ & $\mathrm{x}$ & \\
\hline & \begin{tabular}{|l} 
Informes económicos de empresas \\
Oportunidades de negocio
\end{tabular} & $x$ & 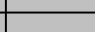 & $\frac{x}{x}$ & & & $x$ & & $x$ & $x$ & $\mathrm{x}$ & & & $x$ & $x$ & & $x$ & $\bar{x}$ & & 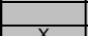 & $x$ & \\
\hline & \begin{tabular}{|l} 
porortuniaasese negocoio \\
Contratación y fiscalidad internacional
\end{tabular} & $x$ & & $\frac{x}{x}$ & & & $x$ & & $x$ & $x$ & & - & & $x$ & $x$ & & $x$ & $x$ & & $x$ & $\frac{x}{x}$ & \\
\hline & Información sobre temas comunitarios & $\mathrm{x}$ & & $x$ & & & & & & 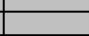 & & & & 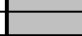 & 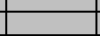 & & & & & $\mathrm{x}$ & $\hat{x}$ & \\
\hline & \begin{tabular}{|l} 
Apoyo Logistico en el exterior \\
\end{tabular} & & & $\frac{x}{x}$ & & & & & & & $x$ & $x$ & & & & & & & & & & \\
\hline & \begin{tabular}{|l|l|l} 
Información sobre programas \\
\end{tabular} & $\frac{x}{x}$ & $x$ & & $x$ & & & & & & \begin{tabular}{|l}
$\mathrm{x}$ \\
$\mathrm{x}$
\end{tabular} & $\frac{x}{x}$ & & $\frac{x}{x}$ & $\frac{x}{x}$ & & $x$ & $x$ & & $x$ & $x$ & \\
\hline & \begin{tabular}{|l} 
Operativa a comercio Exterior \\
Estrategias internacionalización
\end{tabular} & $x$ & $x$ & $x$ & & & & & & $x$ & \begin{tabular}{|c}
$x$ \\
$x$
\end{tabular} & $\frac{x}{x}$ & & $\begin{array}{c}x \\
x \\
x\end{array}$ & $\frac{x}{x}$ & & & & & & & \\
\hline & 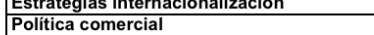 & $\mathrm{x}$ & & $x$ & & & & & & $x$ & \begin{tabular}{|c|c}
$x$ \\
$x$
\end{tabular} & & & & & & & & & & & \\
\hline & Información para empresas extranjeras & $\frac{x}{x}$ & & 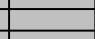 & & & & & & & & - & & $x$ & 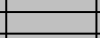 & & & $\mathrm{X}$ & & & - & \\
\hline & \begin{tabular}{|l}
$\mid+\mathrm{D}+\mathrm{i}$ \\
Intelenciacompetitiva
\end{tabular} & \begin{tabular}{|l}
$x$ \\
$x$ \\
$x$
\end{tabular} & $x$ & $x$ & & & & & & & & & & & & & & & & & & \\
\hline \multirow{2}{*}{\multicolumn{2}{|c|}{ 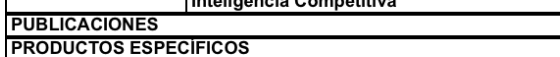 }} & \multirow{2}{*}{\multicolumn{3}{|c|}{$\frac{x}{x}$}} & & & & & & & & & & \multicolumn{3}{|c|}{$x$} & & & & & & \\
\hline & CIFICOS & & & & & & & & & & & & & & $\frac{x}{x}$ & & & $\frac{x}{x}$ & & & & \\
\hline \multirow{2}{*}{\multicolumn{2}{|c|}{ 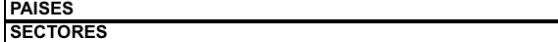 }} & \multirow{2}{*}{\multicolumn{3}{|c|}{$\frac{\frac{x}{x}}{x}$}} & \multirow{2}{*}{\multicolumn{3}{|c|}{$x$}} & \multirow{2}{*}{\multicolumn{3}{|c|}{$\frac{x}{x}$}} & \multirow{2}{*}{\multicolumn{3}{|c|}{$x$}} & \multirow{2}{*}{\multicolumn{3}{|c|}{$\frac{x}{x}$}} & \multicolumn{3}{|c|}{$\frac{x}{x}$} & \multirow{2}{*}{\multicolumn{3}{|c|}{$\frac{x}{x}$}} \\
\hline & & & & & & & & & & & & & & & & & & $\frac{1}{x}$ & & & & \\
\hline & Teléfono & & $\mathrm{SI}$ & & & $\frac{\mathrm{sl}}{\mathrm{SI}}$ & & & $\frac{\mathrm{sl}}{\mathrm{sl}}$ & & & $\frac{\mathrm{SI}}{\mathrm{SI}}$ & & & $\frac{\mathrm{SI}}{\mathrm{SI}}$ & & & $\mathrm{SI}$ & & & $\frac{\mathrm{sl}}{\mathrm{sl}}$ & \\
\hline & \begin{tabular}{|l|l} 
Correo Electrónico \\
Wh/fectricol
\end{tabular} & & $\frac{\mathrm{si}}{\mathrm{si}}$ & & & $\frac{\text { si }}{S 1}$ & & & $\frac{\text { si }}{\text { sit }}$ & & & $\frac{\text { si }}{\text { si }}$ & & & si & & & $\frac{S 1}{S 1}$ & & & $\frac{\mathrm{si}}{\mathrm{si}}$ & \\
\hline CONTACTO & \begin{tabular}{|l} 
Web (formularios) \\
\end{tabular} & & $\mathrm{SI}$ & & & SI & & & SI & & & SI & & & S1 & & & SI & & & SI & \\
\hline & \begin{tabular}{|l|} 
Presencial \\
Redes sociales \\
\end{tabular} & & $\frac{S I}{S i}$ & & & SI & & & SI & & & SI & & & SI & & & $\frac{S I}{S i}$ & & & SI & \\
\hline & \begin{tabular}{|l} 
Contacs Centerter \\
Cont
\end{tabular} & & $\frac{\mathrm{SI}}{\mathrm{NO}}$ & & & NO & & & NO & & & NO & & & NO & & & $\frac{\mathrm{Si}}{\mathrm{NO}}$ & & & SI & \\
\hline INSTRUMENTOS & Sist. Informát. En la gestión de consultas & & SI & & & NO & & & $\mathrm{SI}$ & & & No & & & $\mathrm{SI}$ & & & SI & & & si & \\
\hline PARA LA GESTIÓN & \begin{tabular}{|l|l|} 
CRM \\
\end{tabular} & & $\mathrm{SI}$ & & & SI & & & NO & & & SI & & & NO & & & No & & & $\mathrm{SI}$ & \\
\hline & \begin{tabular}{|l} 
Atención presencial \\
\end{tabular} & & $\frac{S I}{S I}$ & & & $\frac{\mathrm{sl}}{\mathrm{sin}}$ & & & $\frac{S 1}{S I}$ & & & 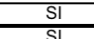 & & & $\frac{S 1}{S !}$ & & & $\frac{S I}{S I+}$ & & & $\frac{S I}{S I I}$ & \\
\hline EL SERVICIO SE & \begin{tabular}{|l} 
De torma centrallazada \\
Distribuida a través de una red de oficinas
\end{tabular} & & $\frac{\mathrm{SI}}{\mathrm{SI}}$ & & & $\frac{s i}{s i}$ & & & $\frac{1}{S 1}$ & & & $\frac{\mathrm{St}}{\mathrm{St}}$ & & & $\frac{\text { si }}{\text { si }}$ & & & $\frac{\mathrm{Sl}}{\mathrm{SI}}$ & & & $\frac{\mathrm{SI}}{\mathrm{SI}}$ & \\
\hline $\begin{array}{l}\text { PRESTA: } \\
\text { PRE }\end{array}$ & $\begin{array}{l}\text { ¿Cuenta con red exterior? } \\
\end{array}$ & & $\frac{\mathrm{SI}}{\mathrm{SI}}$ & & & SI & & & $\frac{1}{s 1}$ & & & $\frac{\mathrm{di}}{\mathrm{d}}$ & & & 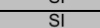 & & & NO & & & si & \\
\hline & Otras redes & & si & & & & & & & & & & & & & & & si & & & & \\
\hline EVALUACIÓN DE & Encuesta de Satisfacción & & SI & & & SI & & & SI & & & SI & & & NO & & & $\frac{\mathrm{SI}}{\mathrm{SI}}$ & & & SI & \\
\hline RESULTADOS & Certificación en Calidad & & SI & & & NO & & & NO & & & SI & & & NO & & & $\mathrm{SI}$ & & & NO & \\
\hline
\end{tabular}




\begin{tabular}{|c|c|c|c|c|c|c|c|c|c|c|c|c|c|c|c|c|c|c|c|c|c|c|}
\hline \multicolumn{23}{|c|}{ CUADRO 5: INSTITUCIONES Y ORGANISMOS A NIVEL REGIONAL (CONTINUACIÓN) } \\
\hline \multicolumn{2}{|l|}{ ORGANISMO } & \multicolumn{3}{|c|}{ INFO MURCIA } & \multicolumn{3}{|c|}{ IPEX } & \multicolumn{3}{|c|}{ IVEX } & \multicolumn{3}{|c|}{ NAVARRA } & \multicolumn{3}{|c|}{ PROEXCA } & \multicolumn{3}{|c|}{ PROMOMADRID (1) } & \multicolumn{3}{|c|}{ SODERCAN } \\
\hline \multirow{18}{*}{$\begin{array}{l}\text { AREAS DE } \\
\text { INFORMACION } \\
\text { ASESORAMIENT } \\
0\end{array}$} & Tipo de información o asesoramiento & $\begin{array}{c}\text { Info. } \\
\text { gratuit } \\
\text { a }\end{array}$ & \begin{tabular}{|c|} 
Asesor \\
a. \\
Gratuito
\end{tabular} & $\begin{array}{l}\text { Asesora. } \\
\text { de Pago }\end{array}$ & \begin{tabular}{|c|} 
Info. \\
gratuit \\
$\mathbf{a}$
\end{tabular} & $\begin{array}{c}\text { Asesor } \\
\text { a. } \\
\text { Gratuito }\end{array}$ & \begin{tabular}{|c|} 
Asesor \\
a. de \\
Pago
\end{tabular} & \begin{tabular}{|c|} 
Info. \\
gratuit \\
a
\end{tabular} & \begin{tabular}{|c|} 
Asesor \\
a. \\
Gratuito
\end{tabular} & $\begin{array}{c}\text { Asesora } \\
\text {.de } \\
\text { Pago }\end{array}$ & \begin{tabular}{|c|} 
Info. \\
gratuita
\end{tabular} & \begin{tabular}{|c|} 
Asesora \\
$\cdot$ \\
Gratuito
\end{tabular} & \begin{tabular}{|c|} 
Asesor \\
a. de \\
Pago
\end{tabular} & $\begin{array}{c}\text { Info. } \\
\text { gratuita }\end{array}$ & $\begin{array}{l}\text { Asesora. } \\
\text { Gratuito }\end{array}$ & $\begin{array}{c}\text { Asesora } \\
\text {.de } \\
\text { Pago }\end{array}$ & \begin{tabular}{|c|} 
Info. \\
gratuit \\
a
\end{tabular} & \begin{tabular}{c|} 
Asesora. \\
Gratuito
\end{tabular} & \begin{tabular}{|c|} 
Asesora \\
. de \\
Pago
\end{tabular} & \begin{tabular}{|c|} 
Info. \\
gratuit \\
a
\end{tabular} & $\begin{array}{l}\text { Asesora. } \\
\text { Gratuito }\end{array}$ & $\begin{array}{c}\text { Asesora } \\
\text {.de } \\
\text { Pago }\end{array}$ \\
\hline & Información país & $\mathrm{x}$ & $\mathrm{x}$ & & $x$ & $\mathrm{x}$ & & $\mathrm{x}$ & & & $\mathrm{x}$ & & & $\mathrm{x}$ & $\mathrm{X}$ & & $\mathrm{x}$ & & & $\mathrm{x}$ & $\mathrm{x}$ & \\
\hline & Para exportar & $\mathrm{x}$ & $\mathrm{x}$ & & $x$ & $x$ & & & & & & & & $x$ & $x$ & & & $\mathrm{x}$ & & $\mathrm{x}$ & $x$ & \\
\hline & Para invertir en el exterior & $\mathrm{X}$ & $\mathrm{x}$ & & & $\mathrm{x}$ & & $\mathrm{x}$ & & & & & & $\mathrm{X}$ & $\mathrm{x}$ & & & $\mathrm{x}$ & & $\mathrm{x}$ & $\mathrm{x}$ & \\
\hline & Estadisticas & $\mathrm{X}$ & $\mathrm{x}$ & & & & & & & & & & & $\mathrm{x}$ & $\mathrm{x}$ & & $\mathrm{x}$ & & & $\mathrm{X}$ & $\mathrm{x}$ & \\
\hline & Contactos comerciales & $\mathrm{x}$ & $\mathrm{x}$ & & & $\mathrm{x}$ & & & & $\mathrm{x}$ & & & $\mathrm{x}$ & $\mathrm{x}$ & $\mathrm{x}$ & & & $\mathrm{x}$ & & $\mathrm{x}$ & $\mathrm{x}$ & \\
\hline & Informes económicos de empresas & & & & $\mathrm{x}$ & & & & & & & & & & & & & & & & & \\
\hline & Oportunidades de negocio & $\mathrm{x}$ & $\mathrm{x}$ & & & $\mathrm{x}$ & & & $\mathrm{x}$ & & & & $x$ & $\mathrm{X}$ & $\mathrm{x}$ & & $\mathrm{x}$ & $\mathrm{x}$ & & $\mathrm{X}$ & $\mathrm{X}$ & \\
\hline & Contratación y fiscalidad internacional & & & & & & & & & & & & & & & & & & & & & \\
\hline & Información sobre temas comunitarios & $\mathrm{x}$ & $\mathrm{x}$ & & & & & & & & & & & & 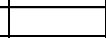 & & & - & & $\mathrm{X}$ & $\mathrm{x}$ & \\
\hline & \begin{tabular}{|l|l} 
Apoyo Logístico en el exterior \\
\end{tabular} & $\mathrm{X}$ & $\mathrm{X}$ & & & & & & & & & & & & & & & & & & & \\
\hline & \begin{tabular}{|l} 
Información sobre programas \\
\end{tabular} & $\mathrm{X}$ & $\mathrm{x}$ & & $\mathrm{x}$ & $\mathrm{x}$ & & $\mathrm{X}$ & & & $\mathrm{x}$ & & & $\mathrm{X}$ & $\mathrm{x}$ & & $\mathrm{X}$ & $\mathrm{x}$ & & $\mathrm{X}$ & $x$ & \\
\hline & Operativa de Comercio Exterior & $x$ & $x$ & & & & & & & & & & & & & & & & & $x$ & $x$ & \\
\hline & Estrategias internacionalización & $\mathrm{x}$ & $\mathrm{x}$ & & & & & & & & & & & & & & & $\mathrm{x}$ & & $\mathrm{x}$ & $x$ & \\
\hline & Política comercial & & & & & & & & & & & & & & & & & & & & & \\
\hline & Información para empresas extranjeras & $\mathrm{x}$ & $\mathrm{x}$ & & $\mathrm{x}$ & $\mathrm{x}$ & & & & & & & & & & & & & & $\mathrm{x}$ & $\mathrm{x}$ & \\
\hline & $1+D+i$ & $\mathrm{X}$ & $\mathrm{X}$ & & & & & & & & & & & & & & & & & $x$ & $x$ & \\
\hline & Inteligencia Competitiva & & & & & & & & & & & & & & & & & & & & & \\
\hline & & & & \multirow{2}{*}{\multicolumn{3}{|c|}{$\frac{x}{x}$}} & & & & & & & & & & & & & \multirow{2}{*}{\multicolumn{3}{|c|}{$x$}} \\
\hline \multirow{2}{*}{\multicolumn{2}{|c|}{\begin{tabular}{|l|} 
PRODUCTOS ESPECIFCOS \\
PAISES
\end{tabular}}} & \multirow{2}{*}{\multicolumn{3}{|c|}{$x$}} & & & & \multirow{2}{*}{\multicolumn{3}{|c|}{$\mathrm{x}$}} & & & & & & & & & & & & \\
\hline \multirow{2}{*}{\multicolumn{2}{|c|}{\begin{tabular}{|l|l|l|l|l|l} 
PAISES \\
SECTORES \\
\end{tabular}}} & & & & \multicolumn{3}{|c|}{$\frac{\hat{x}}{x}$} & & & & & $x$ & & & $x$ & & & $\mathrm{x}$ & & & $x$ & \\
\hline & & & $x$ & & & $x$ & & & $x$ & & & $x$ & & $x$ & & & $x$ & & & & $x$ & \\
\hline & Teléfono & & $\mathrm{SI}$ & & & $\mathrm{SI}$ & & & $\overline{\mathrm{SI}}$ & & & $\overline{\mathrm{SI}}$ & & & $\mathrm{SI}$ & & & $\mathrm{SI}$ & & & $\mathrm{SI}$ & \\
\hline & Correo Electrónico & & SI & & & $\mathrm{SI}$ & & & SI & & & SI & & & SI & & & SI & & & SI & \\
\hline MEDIOS DE & Web (formularios) & & SI & & & NO & & & $\mathrm{SI}$ & & & $\mathrm{SI}$ & & & SI & & & $\mathrm{SI}$ & & & $\mathrm{SI}$ & \\
\hline CONTACTO & Presencial & & $\mathrm{SI}$ & & & $\mathrm{SI}$ & & & $\mathrm{SI}$ & & & $\mathrm{SI}$ & & & $\mathrm{SI}$ & & & $\mathrm{SI}$ & & & $\mathrm{SI}$ & \\
\hline & Redes sociales & & & & & si & & & & & & & & & & & & & & & & \\
\hline & Contact Center & & $\mathrm{NO}$ & & & NO & & & $\mathrm{SI}$ & & & $\mathrm{NO}$ & & & $\mathrm{NO}$ & & & $\mathrm{NO}$ & & & $\mathrm{SI}$ & \\
\hline INSTRUMENTOS & Sist. Informát. En la gestión de & & $\mathrm{SI}$ & & & NO & & & $\frac{\mathrm{SI}}{\mathrm{SI}}$ & & & NO & & & $\mathrm{SI}$ & & & si & & & $\frac{\mathrm{SI}}{\mathrm{SI}}$ & \\
\hline PARA LA & CRM & & NO & & & $\mathrm{SI}$ & & & NO & & & NO & & & NO & & & $\mathrm{SI}$ & & & SI & \\
\hline GESTION & \begin{tabular}{|l|} 
Atención presencial \\
\end{tabular} & & $\mathrm{SI}$ & & & $\mathrm{SI}$ & & & $\mathrm{SI}$ & & & $\mathrm{SI}$ & & & $\mathrm{SI}$ & & & $\mathrm{SI}$ & & & $\mathrm{SI}$ & \\
\hline & De forma centralizada & & $\mathrm{SI}$ & & & NO & & & $\mathrm{SI}$ & & & & & & $\mathrm{SI}$ & & & $\mathrm{SI}$ & & & $\mathrm{SI}$ & \\
\hline EL SERVICIO SE & Distribuida a través de una red de & & & & & $\mathrm{SI}$ & & & $\mathrm{SI}$ & & & & & & SI & & & NO & & & SI & \\
\hline PRESTA: & ¿Cuenta con red exterior? & & $\mathrm{SI}$ & & & $\mathrm{SI}$ & & & $\mathrm{si}$ & & & & & & $\mathrm{SI}$ & & & no & & & $\mathrm{si}$ & \\
\hline & \begin{tabular}{|l|} 
Otras redes \\
\end{tabular} & & & & & & & & & & & & & & & & & si & & & & \\
\hline EVALUACIUIV & Encuesta de Satisfacción & & $\mathrm{SI}$ & & & NO & & & $\mathrm{SI}$ & & & $\mathrm{SI}$ & & & NO & & & $\overline{\mathrm{SI}}$ & & & $\mathrm{SI}$ & \\
\hline DE & Certificcación en Calidad & & NO & & & NO & & & & & & NO & & & NO & & & NO & & & $\mathrm{SI}$ & \\
\hline
\end{tabular}




\section{LOS PROGRAMAS DE PROMOCIÓN DE LA INTERNACIONALIZACIÓN}

Una vez vistos los servicios que ofrecen las distintas OPI en los cuadros anteriores, en este apartado tratamos y agrupamos para que sean entendibles de una mejor manera, así como el tratamiento que ha tenido por distintos autores.

Desde una perspectiva de la empresa, se observa que el desarrollo internacional de estas genera beneficios a corto y medio plazo, y a medio y largo plazo para su país de origen. No obstante, muchas empresas no hacen uso de todo su potencial ni de las oportunidades que ofrecen los mercados exteriores debido a falta de motivación, conocimientos, capacidades, recursos financieros y humanos (Freixanet, 2010). En este sentido, desde el sector público como del privado, se han puesto en marcha una serie de acciones para ayudar a las empresas a superar las barreras que implica su proceso de internacionalización y aumentar así sus posibilidades de éxito en el exterior. En este apartado analizaremos la forma en que las instituciones enunciadas en el epígrafe anterior facilitan el desarrollo económico y social de su región, por medio del apoyo a las empresas locales y permitir que alcancen resultados y competitividad en los mercados internacionales (Gençtürk y Kotabe, 2001).

Es cierto que cada vez más las empresas utilizan los instrumentos de las instituciones de promoción de la internacionalización (Freixanet, 2010), ya que son muchas las que carecen de los conocimientos técnicos adecuados, así como de la formación especializada y experiencia suficiente para acometer en solitario las diferentes actividades que implica su proceso de internacionalización (Moini, 1998). Sin embargo, los programas de promoción no están destinados sólo a cubrir estas carencias, sino que hay otros instrumentos diferentes a los del primer grupo, ya que estos no van encaminados a suplir sus carencias sino a completar los recursos y capacidades.

Otro de los motivos por los que el uso de los instrumentos de promoción de la internacionalización está en aumento es gracias a que las empresas valoran positivamente el apoyo que las instituciones les presta (O’Gorman y Evers, 2011), ya que estas instituciones de fomento de la internacionalización les ofrecen una importante confianza y seguridad, fruto de experiencia y proximidad que tienen hacia estas y gracias a que los instrumentos de apoyo a la internacionalización ofrecidos por las instituciones han ido evolucionando con las necesidades de las empresas (Seringhaus y Botschen, 1991).

Un error muy extendido es identificar internacionalización con exportación. Sin embargo, con este punto de vista se está restringiendo el alcance y objetivos del proceso. Internacionalizar es, desde luego, vender productos y servicios fuera de nuestro país y ésta es la actividad más habitual y la que, en una gran parte de los casos, interesa al empresario. No obstante, internacionalizar es llevar a cabo en un mercado exterior cualquier actividad que pueda ejercer la empresa; internacionalizar es comprar en mercados exteriores, es invertir o asociarse con empresas de otro país, ni siquiera se refiere la internacionalización tan solo a mercancías físicas ya que cada vez tiene más importancia la prestación de servicios, objeto de actividad internacional.

El campo de actuación de la actividad internacional abarca cualquier trabajo que pueda realizar una empresa en el extranjero. Y esto nos lleva a tratar de caracterizar el sujeto de este estudio: la empresa que se internacionaliza. Dentro de las múltiples características que diferencian a las distintas participantes en procesos de internacionalización hay una que es común a la mayoría, hablamos de que generalmente las empresas son de pequeño y mediano tamaño, comúnmente conocidas como pymes. 
Por su propia naturaleza, las pymes presentan ciertas carencias, más acusadas en los procesos de internacionalización, que condicionan la actuación y estructura de los instrumentos de apoyo a la internacionalización. A estas carencias se las conoce como "frenos a la internacionalización". Entre "los frenos" más comunes que se pueden encontrar en la mayoría de las empresas, cabe destacar el desconocimiento de idiomas, poca familiaridad con la legislación local del mercado extranjero al que se dirigen, desconocimiento de los aranceles y otras barreras de entrada, métodos comerciales, información de mercado, etc. Siendo esta la situación de partida de la pyme que se quiere internacionalizar y las entidades públicas se han constituido para apoyarlas a superar los "frenos" a su internacionalización (Wheeler, 1990). En este sentido, dicha ayuda se define como instrumentos de apoyo o promoción de la internacionalización.

La configuración y diseño de estos instrumentos en cualquier institución de promoción viene marcada por las necesidades que las empresas experimentan en su salida y consolidación a los mercados exteriores (Katsikeas, 1996; Seringhaus y Botschen, 1991). La formulación de los mismos se hace sobre la base del uso que la empresa les da a éstos, es decir, se agrupan en virtud del funcionamiento y uso, dando lugar a la siguiente clasificación de instrumentos de apoyo a la internacionalización de:

- Información

- Formación

- Consultoría

- Promoción

- Financiación

- Inversión directa en el extranjero

- Inversión extranjera directa

Estos instrumentos los podemos encontrar en la diversas instituciones con diferentes nombres.

En los próximos apartados, se definirán cada uno de los siete instrumentos y la composición de los mismos.

\section{Información}

Diamantopoulos et al. (2003) propusieron que el grado y las formas en que se utiliza la información de exportación pueden desempeñar un papel significativo en el grado de exportaciones de la empresa, tal y como se observa en la siguiente figura (Toften, 2003).

De esta figura se desprende que la empresa, por medio de la adquisición de información, puede mejorar su rendimiento internacional, sea suya o adquirida (Toften, 2003). Así, la obtención de información adicional permite la reducción de la incertidumbre que rodea a los mercados internacionales, ya sea en un momento dado o de forma continuada en el tiempo (Gillespie y Riddle, 2004; Mcauley 1993; Vyas y Souchon 2003; Wang y Olsen, 2002). La forma en que los programas de exportación facilitan esta adquisición de información son:

- Estudios sectoriales: este servicio ofrece a las empresas información actualizada sobre los mercados a los que se quiere dirigir, concretando la información y según las necesidades (Gençtürk y Kotabe, 2001).

- Bases de datos: este servicio lo usan las instituciones y sirve para generar una base de datos de empresas del entorno a las que ofrecer los servicios de apoyo a la internacionalización y generar acciones de concienciación sobre los mercados exteriores (Gillespie y Riddle, 2004). 
- Seminarios y jornadas técnicas: son seminarios presenciales u on-line que pueden tener diversos formatos, en directo o en diferido ofreciendo información y formación a los empresarios en un breve espacio de tiempo (Seringhaus y Botschen, 1991).

- Servicio de consultas, atención a consultas personalizadas: servicio para ahorrar tiempo a las empresas en la toma de decisiones y quitar incertidumbres en su internacionalización.

- Página WEB, newsletter: es el medio permanente de ofrecer información actualizada y directa a las empresas usuarias de los servicios de las instituciones de promoción de la internacionalización (Francis y Collins-Dodd, 2004) y mantenerlas informadas de sus servicios y de las oportunidades en el extranjero (Gillespie y Riddle, 2004).

- Oportunidades de negocio: Este servicio genera fidelidad de las empresas, ya que las instituciones hacen labores comerciales localizando clientes en el extranjero (Gillespie y Riddle, 2004). Según Bilkey (1978), este es el servicio que se debe ofrecer a las empresas que aún no se han iniciado en la internacionalización para animarlas a iniciar el proceso.

\section{Formación}

Este servicio busca capacitar a los integrantes de las empresas y sus recursos humanos, ya sean trabajadores actuales o futuros, en aspectos vinculados a la internacionalización. Además, con este servicio se trata de involucrar a los no participantes en los procesos de internacionalización a que lo sean (Bilkey, 1978). La forma de realizar este servicio es muy variada y va a depender del presupuesto y de la plantilla con que cuenta la institución de promoción. Los servicios de formación suelen ser los siguientes: becas de internacionalización, cursos de comercio exterior e internacionalización, formación y selección de profesionales internacionales, cátedras de investigación en universidades y convenios con organizaciones de formación.

\section{Consultoría}

La consultoría es un servicio de asesoramiento personalizado a las empresas, con el que se ayuda a dibujar el camino que ha de recorrer la empresa para su internacionalización, en función de sus necesidades o de buscarles los recursos específicos que precisen para la misma. Así, por ejemplo, el asesoramiento en marketing a las empresas mejora las capacidades para su internacionalización (Katsikeas, 1996). Dentro de este instrumento, las instituciones de promoción de la internacionalización suelen ofrecer los siguientes servicios a las empresas:

- Apoyar la generación de Grupos/Consorcios de Exportación: programas para apoyar la salida de forma conjunta de empresas a mercados exteriores.

- Colaboración con Programas Europeos: servicio dirigido a buscar financiación para las empresas o para darles servicios (Seringhaus, 1987).

- Servicio de diagnóstico y plan de acción para la internacionalización: se puede denominar de muchas formas y consiste en el asesoramiento a la empresa por un experto exterior a la misma, coordinado o no por la institución de promoción, y que de forma conjunta con la dirección dibuja el camino hacia la internacionalización de la empresa (Bilkey, 1978). Los distintos nombres que se usan son en base a la empresa usuaria y sus características (Gençtürk y Kotabe 2001), ya que es un servicio personalizado para empresas que comienzan su proceso de internacionalización.

- La consultoría en recursos humanos es un tipo de asesoramiento que ayuda a la profesionalización del equipo directivo y a decidir de forma conjunta, entre instituciones de apoyo y empresas, las necesidades presentes y futuras de las empresas en su internacionalización y las empresas pueden hacerlo con los conocimientos o personas que tenga a su servicio (Johansson y Vahlne, 1977) o adquiriendo nuevos conocimientos y 
recursos, reclutando a profesionales que las instituciones han seleccionado y puesto a disposición de las empresas.

- Servicio de implantación: las empresas una vez que pasa la fase inicial de exportación directa pasan a querer implantarse en un mercado para ofrecer de forma directa sus servicios (Johansson y Vahlne, 1977, 1990), este servicio apoya a las empresas técnica y financieramente a realizar esta implantación.

- Seguro a la exportación: Seringhaus (1987) hablaba de este servicio, que es prestado por las instituciones de promoción de las exportaciones para ayudar a las empresas de su área de competencia a su salida a los mercados exteriores. Las instituciones de promoción contratan una póliza de seguros paragua, a la que se puede unir una empresa que quiera que sus exportaciones, de forma puntual, puedan estar cubiertas de las contingencias acaecidas en los mercados exteriores.

- Consultoría sobre licitaciones multilaterales: las empresas pueden conocer como producir y vender sus servicios o productos. Pero para determinados tipos de venta se requieren unos conocimientos técnicos muy específicos que además precisan de un asesoramiento continuado para poder tener éxito. Este es el caso de las licitaciones multilaterales. Las instituciones de promoción de la internacionalización ofrecen estos servicios a las empresas para licitar a los organismos multilaterales tales como la ONU, Banco Europeo de Reconstrucción y Desarrollo, Banco Interamericano de Desarrollo, Grupo Banco Mundial, etc. (en el apartado anterior se enunciaron y describieron las instituciones multilaterales más importantes).

\section{Promoción}

Este tipo de instrumento lleva la empresa a contactar con interlocutores del mercado objetivo, ya sea para iniciar actividades comerciales, para buscar socio o vender sus productos o servicios directamente y son los instrumentos de apoyo a la internacionalización más deseados por las empresas, al ofrecer los mismos una experiencia directa sobre los mercados (Reid, 1981).

En este instrumento están representados los siguientes servicios de apoyo a la internacionalización:

- Asistencia a ferias internacionales, apoyo a la asistencia a ferias individuales: las ferias son el instrumento de promoción más antiguo para la venta de productos (Hansen, 1996), ya que consiste en asistir a un evento de carácter internacional con otros expositores y allí asisten compradores y vendedores nacionales e internacionales (Wilkinson and Brouthers, 2006), por lo que el producto expuesto es visto por multitud de potenciales compradores en un breve espacio físico (recinto ferial) y de duración.

- Misiones comerciales: este servicio ofrece a un grupo de empresas en un periodo determinado y con uno o varios mercados objetivos, la oportunidad de asistir juntas con fines comerciales (Spence, 2003), ya sean directa, inversa o de estudio.

- Acciones globales de promoción: son acciones de marketing directo para ayudar a la empresa a mostrar su productos en los mercados objetivo (Katsikeas, 1996).

- Participación en promociones de punto de venta: acceder a los grandes lineales de forma temporal para que los consumidores prueben los productos promocionados.

- Convenios con asociaciones, apoyo sectorial e iniciativas agrupadas. La asociación pueden hacer fuerza con la administración para ofrecer servicios de apoyo a la internacionalización de sus asociados (Guillespie y Riddle, 2004; Wheeler, 1990), la institución de promoción conjuntamente con la asociación diseña servicios para los asociados y los financia. 
- Servicio de apoyo a marcas: uno de los objetivos de las empresas puede ser mejorar la imagen de marca en los mercados que ya están presentes (Durmuşoğlu et al., 2012). Con este servicio, la institución de promoción apoya financieramente a las empresas para promocionar su marca en países exteriores.

- Agendas en la red exterior. Este es un instrumento para dar apoyo a las empresas en los propios mercados objetivos de las empresas, los servicios que prestan son de búsqueda de información en el mercado exterior para reducir la incertidumbre de la empresa (Vyas y Souchon, 2003) (Mcauley, 1993), preparación de informes técnicos, localización de facilitadores de entrada y relaciones públicas en esos mercados y proyectos individuales en el exterior. Son servicios de asesoramiento personalizado para un mercado en concreto, se puede decir que son dos instrumentos en uno: asesoramiento y promoción, pues se trata de buscar a una empresa la forma más adecuada de entrar en un mercado $\mathrm{y}$, a su vez, de generarle una agenda con los contactos más idóneos para que tenga éxito y de los primeros pasos en el mercado.

- Participación en campañas sectoriales: actividades de promoción de productos genéricos del área que se promociona: aceite, tomate, aceituna, mueble....y así generar una asociación en los clientes del producto con el origen del mismo.

\section{Financiación}

Este instrumento buscar incentivar por un lado y facilitar por otro la internacionalización de las empresas (Gençtürk y Kotabe, 2001). Las instituciones de promoción de la internacionalización ofrecen apoyo financiero como soporte operativo de las empresas (Seringhaus y Rosson, 1990) y puede tener carácter reembolsable o no reembolsable.

\section{Atracción de inversiones extranjeras}

El enfoque que justifica este instrumento cambia el enfoque utilizado y consiste en crear un entorno favorable para que industrias y empresas extranjeras se establezcan en la región de competencia de la institución de promoción y generen un entorno económico favorable (Porter, 1990).

\section{Promoción de inversiones directas en el extranjero}

Este instrumento busca que las empresas de la zona de competencia de la institución de promoción inviertan en otros mercados y genera valor en estos. La empresa va a invertir en aquellos mercados en los que pueda hacer valer su valor diferencial (Buckley y Hashai, 2009).

\section{CONCLUSIONES Y RECOMENDACIONES}

Por la recopilación de estudios académicos se ve la falta de información por parte de las empresas y por lo ofrecido en este artículo vemos la cantidad de servicios que hay a disposición de las empresas, se concluye que hay que estudiar el conocimiento de los mismos por parte de las empresas y hacer campañas de difusión de los instrumentos entre las empresas.

Así mismo, se concluye que las empresas no han de internacionalizarse de forma autónoma, sino que han de buscar en su entorno apoyo e información de las instituciones que les puedan ayudar a ahorrar tiempo y dinero en su internacionalización.

De todo lo anterior se desprende la existencia de una multitud de servicios a disposición de las empresas y al final serán las necesidades de las empresas las que manden sobre la evolución de los instrumentos ofrecidos por las entidades públicas y el uso que hagan las 
empresas será en función de su utilidad, ahorro de costes y velocidad de llegada a los mercados objetivo.

Sin embargo, y según estudios recientes, el uso y la percepción que las empresas tienen de los organismo de promoción son aún mejorables. Además existe la percepción de que muchos de los esfuerzos que se hacen están duplicados creando mayor confusión para las empresas.

Una obligación de las organizaciones de la promoción de la internacionalización es la de medir la eficacia de sus instrumentos e ir evolucionando los mismos en la medida que lo demandan la economía, los mercados y las empresas, el no hacer esto hará que las empresas sigan con sus procesos de crecimiento, sin contar con estos organismos ni instrumentos, a costa de sus recursos y pérdida de competencia en el mercado, por lo expresado anteriormente.

Ante este panorama tan amplio de instituciones e instrumentos se hace necesaria una clasificación de los instrumentos por grado de internacionalización, necesidades de la empresa, tamaño, mercado objetivo que elimine las incertidumbres de las empresas sobre qué instrumento usar en cada momento, ya que estos pueden estar en distintas instituciones.

Las empresas no deben pararse en la idoneidad o no de los instrumentos sino que los usarán o no en función de sus necesidades, por lo que las instituciones han de evolucionar y adaptar sus ofertas a las necesidades puntuales de las empresas.

El futuro está en la velocidad de adaptación de la oferta a las necesidades y pasa necesariamente por las nuevas tecnologías y la aproximación a las empresas de las facilidades de que se dispone para que accedan a su mercados objetivo, así como una labor de concienciación entre las empresas de la necesidad de internacionalizarse e informarlas del riesgo que corren si lo hacen.

No obstante, los organismos de promoción de internacionalización son los puentes hacia la internacionalización de las empresas de su ámbito, si estos organismos no existiesen, es la propia empresa la que ha de depender de sus propios esfuerzos y capacidades para llegar a los mercados y lo hará pero con mayor dificultad.

La empresas deben ser conscientes de la utilidad que le brindan los instrumentos de apoyo a la internacionalización, ya que ahorran tiempo y dinero, acortando los procesos de internacionalización de las mismas, si una región o país no ofreciera estos instrumentos provocaría un encarecimiento de sus bienes y servicios dejando en desventaja competitiva a las empresas de su órbita económica.

\section{BIBLIOGRAFÍA}

Aldrich, H.E. (1999): Organizations Evolving, Sage.

Ayob, A.H. y Freixanet, J. (2014): "Insights into public export promotion programs in an emerging economy: The case of Malaysian SMEs", Evaluation and Program Planning, 46, pp. 38-46.

Bilkey, W.J. (1978): "An Attempted Integration of the Literature on the Export Behavior of Firms", Journal of International Business Studies, 9(1), pp. 33-46.

Brezzo, R. y Perkal, I. (1983): "The role of marketing incentives in export promotion: The Uruguaian case", en M. Czinkota (Ed.): Export promotion, the public and private sector interaction, New York: Praeger Publishers, pp. 51-65. 
Brossard, H. (1998): "Importance of organizations for investment promotion during an investment decision process: An exploratory study", Management International Review, 38(3), pp. 203-214.

Brouthers, L.E. y Wilkinson, T. (2006): "Trade promotion and SME export performance", International Business Review, 15 (2006), pp. 323-252.

Buckley, P.J. y Hashai, N. (2009): "Formalizing internationalization in the eclectic paradigm", Journal of International Business Studies, 40(1), pp. 58-70.

Cansino, J.M., López-Melendo, J., Pablo-Romero, M.D.P. y Sánchez-Braza, A. (2013): “An economic evaluation of public programs for internationalization: The case of the Diagnostic program in Spain”, Evaluation and Program Planning, 41, pp. 38-46.

Casillas, J.C. y Moreno-Menéndez, A.M. (2014): "Speed of the internationalization process: The role of diversity and depth in experiential learning", Journal of International Business Studies, 45(1), pp. 85-101.

Czinkota, M. R. (1982): Export development strategies, New York, NY: Praeger.

Czinkota, M.R. (2002): "Export promotion: A framework for finding opportunity in change", Thunderbird International Business Review, 3, pp 315.

Diamantopoulos, A., Schlegelmilch, B. B. y Tse, K. Y. (1993): "Understanding the role of export marketing assistance: Empirical evidence and research needs", European Journal of Marketing, 27(4), pp 5-18.

Durmuşoglu, S.S., Apfelthaler, G., Nayir, D.Z., Álvarez, R. y Mughan, T. (2012): “The effect of government-designed export promotion service use on small and medium-sized enterprise goal achievement: A multidimensional view of export performance", Industrial Marketing Management, 41(4), pp. 680-691.

Francis, J y Collins-Dodd, C. (2004): "Impact of export promotion programs on firm competencies, strategies and performance. The case of Canadian high-technology", $21,4 / 5$, pp. 474-495

Freeman, J. y Styles, C. (2014): “Does location matter to export performance?", International Marketing Review, 31(2), pp. 181-208.

Freixanet, J. (2010): "La evaluación del impacto de los programas de promoción de la internacionalización. Una aplicación al caso de las empresas de Cataluña", Información Comercial Española. Revista de Economía, 854, pp. 105-121.

Gençtürk, E.F. y Kotabe, M. (2001): "The effect of export assistance program usage on export performance: A contingency explanation”, Journal of International Marketing, 9(2), pp. 51-72.

Gillespie, K. y Riddle, L. (2004): "Export promotion organization emergence and development: A call to research", International Marketing Review, 21(4), pp. 462-473.

Griffith, D.A., Cavusgil, S.T. y Xu, S. (2008): "Emerging themes in international business research", Journal of International Business Studies, 39(7), pp. 1220-1235.

Hansen, K. (1996): "The dual motives of participants at international trade shows: An empirical investigation of exhibitors and visitors with selling motives", International Marketing Review, 13(2), pp. 39-53.

Hayakawa, K., Lee, H. y Park, D. (2014): “Do export promotion agencies increase exports?", Developing Economies, 52(3), pp. 241-261. 
Johanson, J. y Vahlne, J. (1977): "The internationalization process of the firm--A model of knowledge development and increasing foreign market commitments", Journal of International Business Studies (pre-1986), 8(000001), pp. 23.

Johanson, J. y Vahlne, J. (1990): “The Mechanism of Internationalization", International Marketing Review, 7(4), pp. 11.

Katsikeas, C.S. (1996): "Ongoing export motivation: Differences between regular and sporadic exporters", International Marketing Review, 13(2), pp. 4-19.

Kotabe, M. y Czinkota, M.R. (1992): "State government promotion of manufacturing exports: A gap analysis”, Journal of International Business Studies, 23(4), pp. 637.

Lemaghen, F. (1987): "A pilot evaluation of the export marketing research scheme, for Richard Young”, British Overseas Trade Board, Schanmark Export Marketing Services.

Mcauley, A. (1993): “The perceived usefulness of export information sources", European Journal of Marketing, 27(10), pp. 52.

Moini, A.H. (1998): "Small firms exporting: How effective are government export assistance programs?", Journal of Small Business Management, 36(1), pp. 1-15.

O'Gorman, C. y Evers, N. (2011): "Network intermediaries in the internationalization of new firms in peripheral regions", International Marketing Review, 28(4), pp. 340-364.

Porter, M. (1990): The Competitive Advantage of Nations, New York: Free Press.

Reid, S.D. (1981): "The decision-maker and export entry and expansion", Journal of International Business Studies (pre-1986), 12(000002), pp. 101.

Seringhaus, F.H.R. (1986): "The impact of government export marketing assistance", International Marketing Review, 3(2), pp. 61-72.

Seringhaus, F.H.R. (1987): "Export promotion: the role and impact of government services", International Marketing Review, Vol. 2, pp. 106-116.

Seringhaus, F.H.R. y Botschen, G. (1991): "Cross-National Comparison of Export Promotion Services: The views of Canadian and Austrian companies", Journal of International Business Studies, 22(1), pp. 115.

Seringhaus, F.H.R. and Rosson, P.J. (1990): Government export promotion: A global perspective, International Business Series London and New York: Routledge.

Spence, M.M. (2003): "Evaluating export promotion programs: U.K. overseas trade missions and export performance", Small Business Economics, 20(1), pp. 83.

Toften, K. y Svein, O.O. (2003): "Export market information use, organizational knowledge, and firm performance: A conceptual framework", International Marketing Review, 20(1), pp. 95.

Vyas, R. y Souchon, A.L. (2003) "Symbolic use of export information: A multidisciplinary approach to conceptual development and key consequences", International Marketing Review, 20(1), pp. 67-

Wang, G. y Olsen, J. E. (2002): "Knowledge, performance, and exporter satisfaction: An exploratory study", Journal of Global Marketing, vol. 15 (3/4), pp. 39-64.

Wheeler, C.N. (1990): "Stimulating Scottish and United Kingdom economies through export promotion programs", in Cavusgil, S.T. and Czinkota, M.R. (Eds), International Perspectives on Trade. 
Wilkinson, T.J. (2006): "Entrepreneurial Climate and U.S. State Foreign Trade Offices as Predictors of export success", Journal of Small Business Management, 44(1), pp. 99113. 